Nota científica

\title{
Registros nuevos de Orchidaceae para el estado de Tabasco, México
}

\author{
New records of Orchidaceae for the state of Tabasco, Mexico \\ Jonas Morales-Linares ${ }^{\mathrm{a}, *}$, Tarin Toledo-Aceves ${ }^{\mathrm{a}}$, Alejandro Flores-Palacios ${ }^{\mathrm{b}}$, \\ Thorsten Krömer ${ }^{\mathrm{c}}$ y José G. García-Franco ${ }^{\mathrm{a}}$ \\ ${ }^{a}$ Red de Ecología Funcional, Instituto de Ecología, A. C., Carretera antigua a Coatepec 351, El Haya, 91070, Xalapa, Veracruz, México \\ ${ }^{\mathrm{b}}$ Centro de Investigación en Biodiversidad y Conservación, Universidad Autónoma del Estado de Morelos. Av. Universidad 1001, Col. Chamilpa, 62209, \\ Cuernavaca, Morelos, México \\ ${ }^{\mathrm{c}}$ Centro de Investigaciones Tropicales, Universidad Veracruzana, Exhacienda Lucas Martín Privada de Araucarias s. n., Col. Periodistas, 91019, Xalapa, \\ Veracruz, México
}

Recibido el 29 de septiembre de 2014; aceptado el 26 de mayo de 2015

Disponible en Internet el 27 de octubre de 2015

\begin{abstract}
Resumen
Se documentan 7 nuevos registros de orquídeas para la flora del estado de Tabasco, México. La distribución de cada especie fue evaluada para México y el resto del Neotrópico mediante información obtenida de listados florísticos, registros de herbario y herramientas en línea. Finalmente, se discute la importancia del bosque tropical perennifolio como hábitat para la conservación de las orquídeas en un estado severamente alterado por deforestación y cambios en el uso de suelo como Tabasco.

Derechos Reservados ( 2015 Universidad Nacional Autónoma de México, Instituto de Biología. Este es un artículo de acceso abierto distribuido bajo los términos de la Licencia Creative Commons CC BY-NC-ND 4.0.
\end{abstract}

Palabras clave: Coryanthes picturata; Corymborkis forcipigera; Gongora truncata; Lycaste cochleata; Mormolyca hedwigiae; Ornithocephalus bicornis; Orquídeas; Stanhopea dodsoniana

\section{Abstract}

Seven orchid species are reported as new records for the state of Tabasco, Mexico. The distribution of each species was evaluated for Mexico and the rest of the Neotropics considering data of floristic inventories, herbarium records and online tools. Finally, the importance of the tropical rain forest as habitat for orchid conservation in a severely altered state by deforestation and land use changes as Tabasco is discussed.

All Rights Reserved @ 2015 Universidad Nacional Autónoma de México, Instituto de Biología. This is an open access item distributed under the Creative Commons CC License BY-NC-ND 4.0.

Keywords: Coryanthes picturata; Corymborkis forcipigera; Gongora truncata; Lycaste cochleata; Mormolyca hedwigiae; Ornithocephalus bicornis; Orchids; Stanhopea dodsoniana

En México, como en otras regiones neotropicales, las orquídeas son muy diversas en los bosques mesófilos de montaña y en los bosques tropicales perennifolios (Gentry y Dodson, 1987; Hágsater et al., 2005; Kreft, Köster, Küper, Nieder y Barthlott, 2004). Actualmente, la orquideoflora mexicana incluye aproximadamente 1,250 especies y 168 géneros, y aunque está bien documentada (Hágsater et al., 2005; Soto-Arenas

\footnotetext{
* Autor para correspondencia.

Correo electrónico: jonasm11 @gmail.com (J. Morales-Linares).

La revisión por pares es responsabilidad de la Universidad Nacional Autónoma de México.
}

et al., 2007), en años recientes se han descrito varias especies nuevas (por ejemplo, Carnevali, Cetzal-Ix, Balam-Narváez y Romero-González, 2010; Cetzal-Ix y Carnevali, 2010; Ramos-Castro, Castañeda-Zárate, Solano-Gómez y Salazar, 2012; Salazar, Chávez-Rendón, Jiménez-Machorro y De Ávila, 2011; Salazar, Hernández-Cardona y Jiménez-Machorro, 2013; Solano-Gómez, 2010; Soto-Arenas y Salazar, 2012;), y reportado registros nuevos de algunas especies (por ejemplo, Salazar, 2013; Solano-Gómez, Jiménez-Machorro y Damon, 2011; Solano-Gómez y Martínez-Ovando, 2011). Por ejemplo, en estados como Veracruz (Castañeda-Zárate, Viccon-Esquivel, Ramos-Castro y Solano-Gómez, 2012; Castillo-Campos, 


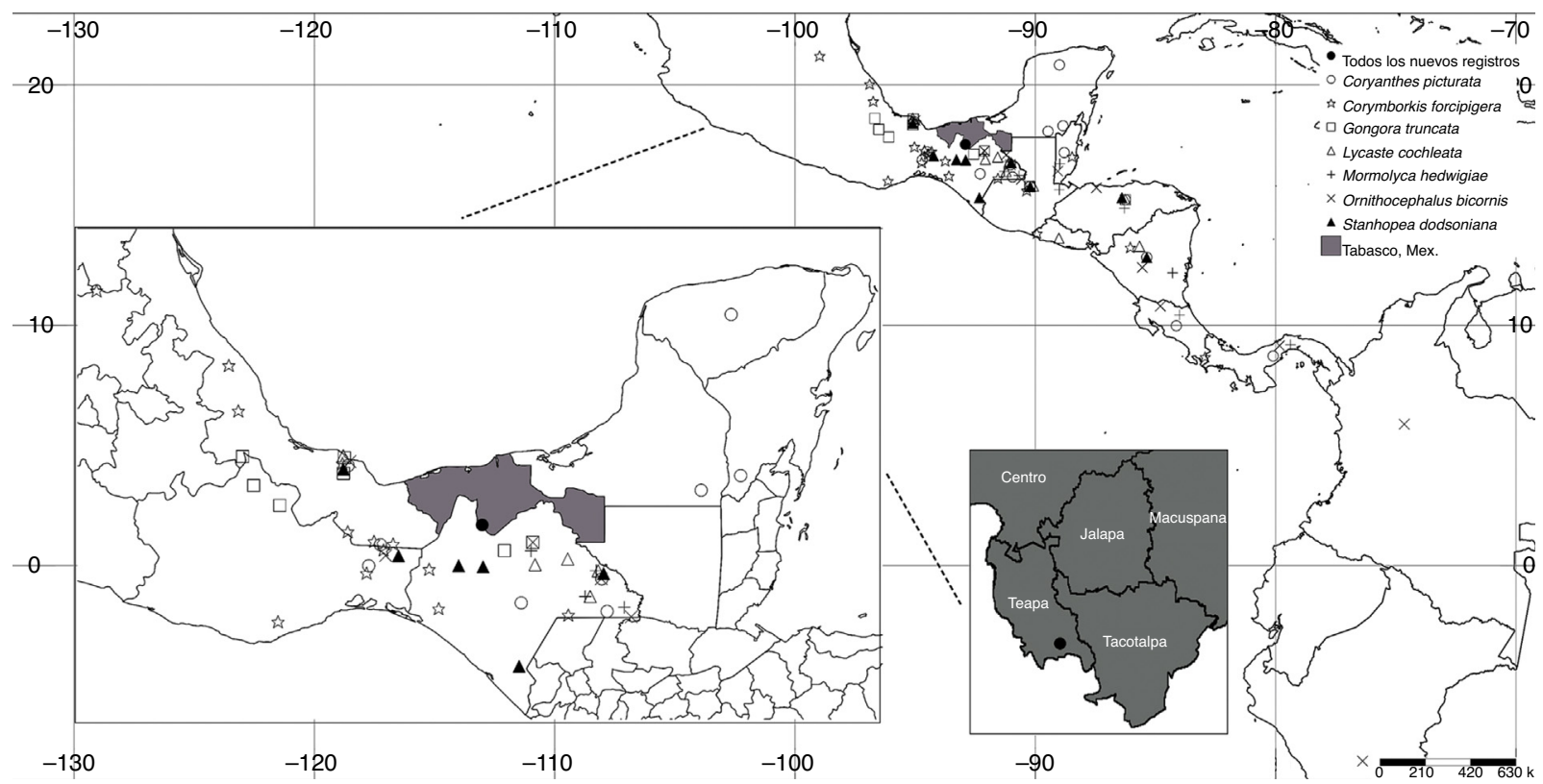

Figura 1. Ubicación de los nuevos registros de orquídeas para el estado de Tabasco, México y su distribución en el Neotrópico. La distribución de las especies en países distintos a México se representa con un símbolo por país, aunque generalmente tienen un mayor número de registros.

García-Franco, Mehltreter y Martínez, 2009) y Tabasco (Noguera-Savelli y Cetzal-Ix, 2013, 2014) se han documentado registros nuevos, lo que se sugiere que todavía existen sitios por explorar y en ellos especies por descubrir y reportar.

Para Tabasco, el conocimiento de las Orchidaceae se basa en las publicaciones de Alderete-Chávez (1992); Alderete-Chávez y Cappello-García (1988), algunos inventarios florísticos (por ejemplo Cruz-Hernández, 2000; Pérez, Sousa, Hanan, Chiang y Tenorio, 2005), y la revisión actualizada de Noguera-Savelli y Cetzal-Ix (2014), quienes reportan para el estado un total de 109 especies de orquídeas y una variedad distribuidas en 58 géneros. Por otro lado, Tabasco es uno de los estados más deforestados de México, entre los años 1950-1991 perdió el 92.4\% de la superficie de sus bosques debido al desarrollo de la agricultura y la ganadería (Sánchez-Munguía, 2005). En el centro-sur del estado, en municipios como Teapa y Tacotalpa, aún se encuentran remanentes de bosque tropical perennifolio, hábitat de la mayoría de las orquídeas de Tabasco (CruzHernández, 2000).

En 2011 iniciamos una evaluación de la diversidad de orquídeas en el bosque tropical perennifolio de la sierra Madrigal, en donde se encuentra el Parque Estatal de la Sierra, municipio de Teapa; así como en la vegetación ribereña, plantaciones de cacao bajo sombra (Morales-Linares, 2012) y pastizales para ganadería de los alrededores. Durante el proceso de identificación de las especies registradas también se evaluó su distribución en México con información obtenida de listados especializados (Espejo-Serna y López-Ferrari, 1997, 1998; Noguera-Savelli y Cetzal-Ix, 2014; Soto-Arenas, 1988) y de ejemplares de herbario tanto nacionales (MEXU, XAL y AMO) como extranjeros (MO) disponibles en bases de datos en línea (GBIF, 2014; Tropicos, 2014). Basados en el análisis de la información anterior, se proponen 7 especies como nuevos registros de Orchidaceae para Tabasco (figs. 1 y 2). Adicionalmente, se incluye la distribución de cada especie en el resto del Neotrópico considerando un registro de herbario por país (fig. 1). El mapa de distribución de las especies fue elaborado en línea (Shorthouse, 2010).

Coryanthes picturata Rchb. f., Botanische Zeitung (Berlín) 22(43): 332.1864 (fig. 2A).

Ejemplares de referencia. México: Campeche: D. Álvarez 5994 (MEXU); Chiapas: E. Martínez et al., 25795 (AMO) y G. Aguilar 6269 (MEXU); Oaxaca: H. Hernández 1272 (CHAPA); Quintana Roo: O. Blanco et al., 5483 (CICY); Tabasco: J. Morales-Linares 99 (XAL); Veracruz: G. Salazar et al., 5511 (MEXU) y S. Sinaca 1258 (MEXU); Yucatán: G. Carnevali 6377 (CICY). Belice: P. Catling B90-18 (AMES); Costa Rica: C. Horich O-8991 (HEID). Guatemala: H. Türckheim 7680 (US). Honduras: J. Edwards 649 (AMES). Nicaragua: H. G. Seeger O-21275 (M). Panamá: E. Olmos et al., 02/2948 (M).

Distribución y hábitat. C. picturata se distribuye desde el sur de México hasta Panamá (fig. 1). En Tabasco, esta orquídea epífita habita frecuentemente en vegetación ribereña, en el borde del bosque tropical perennifolio y de manera muy importante en plantaciones de cacao y naranja.

Observaciones. Las especies del género Coryanthes se caracterizan por una morfología floral muy compleja y por estar asociadas con nidos arbóreos de hormigas denominados jardines de hormigas (Gerlach, 2011).

Corymborkis cf. forcipigera (Rchb. f. y Warsz.) L. O. Williams, Botanical Museum Leaflets 12(7): 237. 1946 (fig. 2B).

Ejemplares de referencia. México: Chiapas: D. E. Breedlove 24108, 38001 y 56584 (MO); Hidalgo: R. Mayorga y O. Alcántara 868 (FCME); Oaxaca: G. Salazar 5535 (AMO), H. Hernández 2385 (CHAPA) y K. Velasco 273 (MEXU); Tabasco: J. Morales-Linares 83 (XAL) y 217 (en cultivo); Veracruz: A. Gómez 277 (XAL), C. Purpus 7509 (XAL), M. Vázquez 979 (XAL), R. Acosta y C. Juárez 1403 (AMO), S. Sinaca 1045 

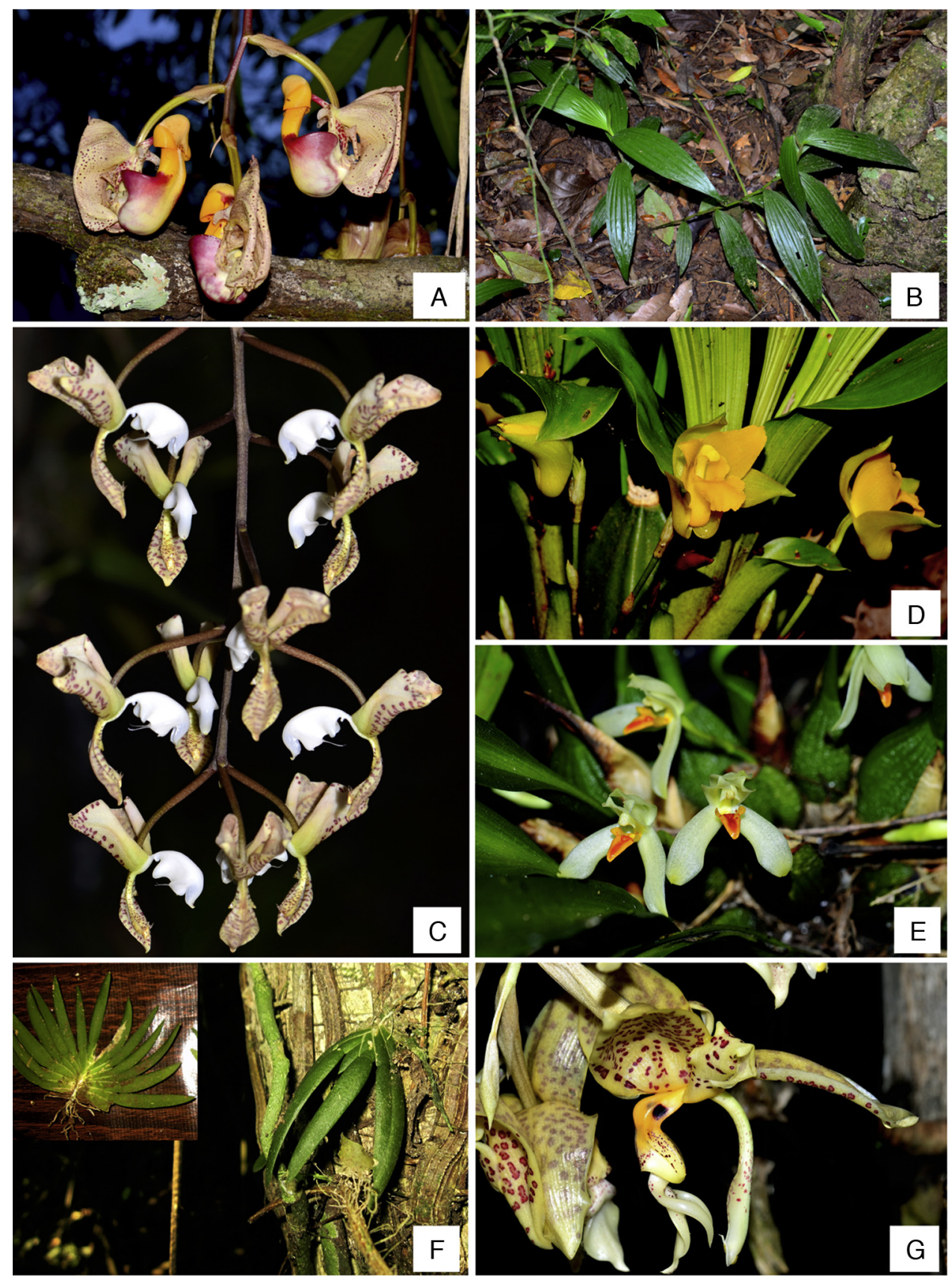

Figura 2. Especies de orquídeas que son nuevos registros para el estado de Tabasco, México. A) Flores de Coryanthes picturata. B) Plantas de Corymborkis cf. forcipigera. C) Inflorescencia de Gongora truncata. D) Flores de Lycaste cochleata. E) Flores de Mormolyca hedwigiae. F) Plantas de Ornithocephalus bicornis. G) Flores de Stanhopea dodsoniana. Fotografías J. Morales-Linares.

(MEXU) y T. Wendt 4021 (MEXU). Belice: W. Schipp 302 (F). Costa Rica: B. Hammel y M. Grayum 14189 (MO). El Salvador: R. Villacorta 850 (MO). Guatemala: J. Steyermark 44989 (F). Nicaragua: I. Coronado et al., 5611 (HULE).

Distribución y hábitat. C. forcipigera se ha registrado desde México hasta Costa Rica (fig. 1), y también se ha documentado su presencia en Colombia y en islas del Caribe (GBIF, 2014; Tropicos, 2014). Esta orquídea terrestre se encontró habitando sitios muy sombreados del interior del bosque tropical perennifolio en poblaciones aisladas de pocos individuos.

Observaciones. Esta especie puede ser confundida con algunas palmas del sotobosque (Chamaedorea spp.), ya que vegetativamente son muy semejantes. No se pudo localizar algún individuo con estructuras reproductivas, pero su altura mayor a $50 \mathrm{~cm}$ y tallos no ramificados la diferencian de Tropidia polystachya (Sw.) Ames, especie de orquídea muy similar (Ames y Correll, 1985).

Gongora truncata Lindl., Edwards's Botanical Register 29: Misc. 42.1843 (fig. 2 C).

Ejemplares de referencia. México: Chiapas: A. Shilom 7203 (MO) y R. Leleu s.n. (AMO); Oaxaca: J. I. Calzada 10343 (XAL) y R. Torres 11081 (MEXU); Tabasco: J. Morales-Linares 84 (en cultivo) y 233 (XAL); Veracruz: O. Nagel 4108 (XAL), R. Cedillo 3074 (MEXU) y T. Krömer et al., 1909 (MEXU). 
Guatemala: M. A. Dix 9448 (UVAL). Honduras: P. Allen 6044 (SEL).

Distribución y hábitat. G. truncata se distribuye en el sureste de México, Guatemala y Honduras (fig. 1), pero también se ha documentado su presencia en Belice (GBIF, 2014; Tropicos, 2014). Esta orquídea epífita se encontró en el bosque tropical perennifolio; sus poblaciones se encuentran dispersas y tienen pocos individuos, los cuales crecen preferentemente sobre el fuste de sus árboles hospederos.

Observaciones. Esta especie es similar a Gongora leucochila Lem., y Gongora unicolor Schltr., pero se distingue de ellas por sus flores con ovarios divaricados (Salazar, 1990a) y sus pétalos más cortos (longitud) en relación con la columna.

Lycaste cochleata Lindl., Paxton's Flower Garden 1: 126. 1853[1850] (fig. 2D).

Ejemplares de referencia. México: Chiapas: A. Durán y S. Levy 592 (MEXU), A. Reyes 2256 (MEXU), D. Álvarez 2471 (MEXU) y E. Martínez 15636 (XAL); Tabasco: J. MoralesLinares 88 (XAL); Veracruz: S. Sinaca 63 (MEXU). El Salvador: E. Ávila 321 (MO). Guatemala: J. Steyermark 42960 (AMES). Honduras: A. Molina 7165 (EAP). Nicaragua: A. Heller 9426 (SEL).

Distribución y hábitat. L. cochleata se encuentra desde el sureste de México hasta Nicaragua (fig. 1). Un solo individuo de esta orquídea epífita se encontró en el interior del bosque tropical perennifolio.

Observaciones. La flor presenta en el labelo lóbulos laterales más grandes y ápices más redondos que los que presenta Lycaste aromatica (Graham) Lindl., especie morfológicamente semejante (Salazar, 1990b), y que hasta ahora era la única del género reportada para Tabasco (Noguera-Savelli y Cetzal-Ix, 2014).

Mormolyca hedwigiae (Hamer y Dodson) M. A. Blanco, Lankesteriana 7(3): 531.2007 (fig. 2E).

Ejemplares de referencia. México: Chiapas: D. E. Breedlove 33203 (CAS), D. E. Breedlove y F. Almeda 48292 (CAS), E. Martínez 16518 (MO) y G. Aguilar 9076 (MEXU); Tabasco: J. Morales-Linares 186 (XAL). Belice: T. Hawkins 1070 (MO). Costa Rica: J. Folsom 9448 (DUKE). Guatemala: F. Hamer A161 (MO). Honduras: P. Allen 3879 (SEL). Nicaragua: D. Soza et al., 356 (MO). Panamá: R. Dressler 3305 (MO).

Distribución y hábitat. M. hedwigiae está presente desde el sureste de México hasta Panamá (fig. 1), aunque también se ha documentado para Colombia (GBIF, 2014; Tropicos, 2014). En México solo se había registrado en Chiapas (selva Lacandona) y se esperaba su presencia en Tabasco (Pérez et al., 2005). En el sitio de registro solo se encontraron 3 individuos creciendo en un árbol aislado de un pastizal ganadero.

Observaciones. En la región, esta orquídea es relativamente fácil de reconocer, ya que despliega un pedúnculo corto y las flores solitarias son color blanquecino con el labelo anaranjado (Soto-Arenas, 1986).

Ornithocephalus bicornis Lindl. ex Benth., The Botany of the Voyage of H. M. S. Sulphur 172. 1844 [1846] (fig. 2F).

Ejemplares de referencia. México: Chiapas: E. Hágsater y R. Triay 4008 (AMO), E. Martínez 15958 (MEXU) y G. Aguilar y C. Chancayun 5489 (MEXU); Oaxaca: G. Salazar y
E. Hágsater registro visual (Salazar y Hágsater, 1997); Tabasco: E. Martínez 34713 (MEXU) y J. Morales-Linares 94 (en cultivo); Veracruz: G. Salazar 481 (MEXU). Belice: W. Schipp S-839 (AMES). Costa Rica: M. Grayum et al., 9058A (MO). Guatemala: E. Contreras 3501 (LL). Honduras: J. MacDougal et al., 3353 (MO). Nicaragua: A. Grijalva y W. Robleto 2265 (MO). Panamá: T. Croat 8223 (MO). Colombia: A Cogollo y R. Borja 1600 (MO). Perú: T. Croat 57986A (MO).

Distribución y hábitat. O. bicornis tiene el mayor intervalo de distribución de las especies aquí reportadas, se encuentra desde el sureste de México hasta Perú (fig. 1). Asimismo, también se conoce de Brasil, Ecuador, Guayana Francesa, Guyana, Surinam y Venezuela (GBIF, 2014; Tropicos, 2014). Esta orquídea epífita ya se había recolectado en Teapa, Tabasco (E. Martínez 34713); sin embargo, no se había considerado en los trabajos previamente publicados de la orquideoflora del estado. El ejemplar complementario (J. Morales-Linares 94) se encontró en el interior del bosque tropical perennifolio y solo 3 individuos juveniles fueron contabilizados.

Observaciones. Esta especie se distingue de sus congéneres porque presenta inflorescencias lanuginosas, vainas foliares largas (Ames y Correll, 1985) y raíces glabras (G. Salazar, com. pers.).

Stanhopea dodsoniana Salazar y Soto Arenas, Lindleyana 16(3): 144-147, f. 1, 2B. 2001 (fig. 2G).

Ejemplares de referencia. México: Chiapas: E. Hágsater 1393 (AMO), E. Martínez y M. Soto 5226 (AMO), E. Martínez et al., 5715 (AMO) y G. Salazar y M. Soto 1726 (AMO); Oaxaca: M. Soto et al., 7930 (AMO); Tabasco: J. Morales-Linares 92 (XAL); Veracruz: G. Salazar 2137 (AMO). Guatemala: H. Türckheim s.n. (MO). Honduras: J. Edwards 52 (MO). Nicaragua: G. Krönlein et al., 01/409 (MO).

Distribución y hábitat. S. dodsoniana se ha reportado de México, Guatemala, Honduras y Nicaragua (fig. 1). Esta orquídea epífita se encontró en el interior del bosque tropical y es muy escasa.

Observaciones. S. dodsoniana es similar a Stanhopea oculata (G. Lodd.) Lindl., y a Stanhopea whittenii Soto-Arenas, Salazar y G. Gerlach; sin embargo, las 3 especies se distinguen porque $S$. dodsoniana tiene flores desde color crema hasta amarillo-anaranjado y un hipoquilo giboso, $S$. oculata posee flores blanquecinas y un hipoquilo redondeado y $S$. whittenii se caracteriza por su hipoquilo corto y masivo (Salazar y SotoArenas, 2003; Soto-Arenas, 2003).

Las 7 especies registradas en este trabajo por primera vez para Tabasco presentan una distribución amplia, incluyendo estados del sureste de México y varios países del Neotrópico. En particular para México, los sitios en los cuales se han encontrado la mayoría de estas orquídeas corresponden a los bosques tropicales perennifolios de Los Tuxtlas (Veracruz), Los Chimalapas-Uxpanapa (Oaxaca-Veracruz), la selva Lacandona (Chiapas) y la sierra Madrigal (Tabasco-Chiapas) en donde se encuentra el Parque Estatal de la Sierra, regiones que históricamente estuvieron conectadas por bosques tropicales prácticamente continuos desde el norte del estado de Veracruz hasta el estado de Quintana Roo (Rzedowski, 2006). En este sentido, la conservación de estas regiones es prioritaria debido 
a que albergan una gran diversidad de especies, pero también están muy expuestas a la deforestación generada por diversas actividades antrópicas (Dirzo, Aguirre y López, 2009).

Ninguna de estas 7 especies se encuentra en alguna categoría de riesgo a nivel nacional (Nom-059-Ecol-2010, Semarnat, 2010); sin embargo, su presencia en Tabasco es muy reducida en extensión y en número de individuos, por lo que localmente podrían estar en peligro. Aunado a ello, en Tabasco la tasa de deforestación es particularmente alta (Sánchez-Munguía, 2005), por ello es importante continuar documentando la diversidad de orquídeas y otras especies que se encuentren en los fragmentos de bosque tropical perennifolio y demás tipos de vegetación de su territorio. Un ejemplo de lo anterior es $C y c$ noches ventricosum Bateman, especie amenazada (Semarnat, 2010), recientemente registrada para Tabasco de los municipios de Tacotalpa (Noguera-Savelli y Cetzal-Ix, 2013) y Teapa (J. Morales-Linares 185, XAL), la cual aunque generalmente se establece sobre troncos en descomposición en claros del bosque tropical (Soto-Arenas y Salazar, 2007), también se ha encontrado en árboles de cacao y otros más usados para dar sombra dentro de plantaciones de cacao (Morales-Linares, 2012). De este modo, también es importante realizar más exploraciones botánicas en los agroecosistemas (por ejemplo, cacao, cítricos y hule), las cercas vivas y los árboles aislados en pastizales ganaderos. Finalmente, las herramientas de mapeo y ejemplares de herbario en línea (GBIF, 2014; Shorthouse, 2010; Tropicos, 2014) facilitaron la evaluación de la distribución de las orquídeas registradas, por lo que su uso también podría robustecer y agilizar las estrategias para la conservación de estas y otras especies.

Agradecemos el apoyo en campo de Andrés MartínezBaldivia y los comentarios de Gerardo Salazar acerca de la identidad de las especies. Las sugerencias de 2 revisores anónimos contribuyeron a mejorar el manuscrito. El primer autor, agradece al Conacyt por la beca de maestría (\#250340) y al Inecol por la instrucción científica y el respaldo brindado (\#20030-10144).

\section{Referencias}

Alderete-Chávez, A. (1992). Listado complementario de las orquídeas de Tabasco. Agrociencia, Serie Recursos Naturales Renovables, 2, 106-123.

Alderete-Chávez, A. y Cappello-García, S. (1988). Orquídeas de Tabasco. Villahermosa: Gobierno del Estado de Tabasco. Instituto Nacional de Investigaciones sobre Recursos Bióticos.

Ames, O. y Correll, D. S. (1985). Orchids of Guatemala and Belize. Nueva York: Dover Publications, Inc.

Carnevali, G., Cetzal-Ix, W. R., Balam-Narváez, R. y Romero-González, G. A. (2010). A synopsis of Cohniella (Orchidaceae Oncidiinae). Brittonia, 62, 153-177.

Castañeda-Zárate, M., Viccon-Esquivel, J., Ramos-Castro, S. E. y SolanoGómez, R. (2012). Registros nuevos de Orchidaceae para Veracruz, México. Revista Mexicana de Biodiversidad, 83, 281-284.

Castillo-Campos, G., García-Franco, J. G., Mehltreter, K. y Martínez, M. L. (2009). Registros nuevos de Ponthieva brenesii (Orchidaceae) y Piper xanthostachyum (Piperaceae) para el estado de Veracruz, México. Revista Mexicana de Biodiversidad, 80, 565-569.

Cetzal-Ix, W. R. y Carnevali, G. (2010). A revision of Cohniella Pfitzer (Orchidaceae) in Mexico. The Journal of the Torrey Botanical Society, 137, 180-213.

Cruz-Hernández, J. A. (2000). Las orquídeas de Tabasco y sus afinidades ecológicas. Tesis. Texcoco: Universidad Autónoma Chapingo.
Dirzo, R., Aguirre, A. y López, J. C. (2009). Diversidad florística de las selvas húmedas en paisajes antropizados. Investigación Ambiental, 1, $17-22$.

Espejo-Serna, A. y López-Ferrari, A. R. (1997). Las monocotiledóneas mexicanas: una sinopsis florística. 1. Lista de referencia. Parte VII. Orchidaceae 1. México, D. F.: Consejo Nacional de la Flora de México, A. C., Universidad Autónoma Metropolitana Iztapalapa, Comisión Nacional para el Conocimiento y Uso de la Biodiversidad.

Espejo-Serna, A. y López-Ferrari, A. R. (1998). Las monocotiledóneas mexicanas: una sinopsis florística. 1. Lista de referencia. Parte VIII. Orchidaceae 2. México, D. F.: Consejo Nacional de la Flora de México, A. C., Universidad Autónoma Metropolitana Iztapalapa, Comisión Nacional para el Conocimiento y Uso de la Biodiversidad.

GBIF (Global Biodiversity Information Facility). (2014). Recuperado el 9 septiembre, 2014 de: <http://www.gbif.org

Gentry, A. H. y Dodson, C. H. (1987). Diversity and biogeography of neotropical vascular epiphytes. Annals of the Missouri Botanical Garden, 74, $205-233$.

Gerlach, G. (2011). The genus Coryanthes: a paradigm in ecology. Lankesteriana, $11,253-264$.

Hágsater, E., Soto-Arenas, M. A., Salazar, G., Jiménez-Machorro, R., López, M. y Dressler, R. (2005). Las orquídeas de México. México, D. F: Instituto Chinoín, A. C.

Kreft, H., Köster, N., Küper, W., Nieder, J. y Barthlott, W. (2004). Diversity and biogeography of vascular epiphytes in Western Amazonia, Yasuní, Ecuador. Journal of Biogeography, 31, 1463-1476.

Morales-Linares, J. (2012). Diversidad y conservación de orquídeas en plantaciones de cacao del sureste de México. Tesis de maestría. Xalapa: Instituto de Ecología, A. C.

Noguera-Savelli, E. y Cetzal-Ix, W. R. (2013). Primer registro de Cycnoches ventricosum (Orchidaceae: Catasetinae) para Tabasco, México. Botanical Sciences, 91, 233-236.

Noguera-Savelli, E. y Cetzal-Ix, W. R. (2014). Revisión e integración del conocimiento de las Orchidaceae de Tabasco, México. Botanical Sciences, 92, 519-540.

Pérez, L. A., Sousa, M., Hanan, A. M., Chiang, F. y Tenorio, P. (2005). Vegetación terrestre. En J. Bueno, F. Álvarez y S. Santiago (Eds.) Biodiversidad del estado de Tabasco (pp. 65-110). México, D. F.: Instituto de Biología, Universidad Nacional Autónoma de México, Comisión Nacional para el Conocimiento y Uso de la Biodiversidad.

Ramos-Castro, S. E., Castañeda-Zárate, M., Solano-Gómez, R. y Salazar, G. A. (2012). Stelis zootrophionoides (Orchidaceae: Pleurothallidinae), a new species from Mexico. PLoS ONE, 7, e48822. http://dx.doi.org/10.1371/journal. pone.0048822.

Rzedowski, J. (2006). Vegetación de México. México, D. F: Comisión Nacional para el Conocimiento y Uso de la Biodiversidad.

Salazar, G. A. (1990a). Gongora truncata Lindl. En E. Hágsater y G. Salazar (Eds.), Icones Orchidacearum 1. Orchids of Mexico (1) (p. 45). México, D. F: Asociación Mexicana de Orquideología, A. C.

Salazar, G. A. (1990b). Lycaste cochleata Lindl. En E. Hágsater y G. Salazar (Eds.), Icones Orchidacearum 1. Orchids of Mexico. (1) (p. 57). México, D. F: Asociación Mexicana de Orquideología, A. C.

Salazar, G. A. (2013). Two additions to the Mexican orchid flora. Revista Mexicana de Biodiversidad, 84, 378-380.

Salazar, G. A. y Hágsater, E. (1997). Diversidad y conservación de orquídeas de la región de Chimalapa, Oaxaca, México. Reporte final del proyecto $G$ 024. México, D. F.: Comisión Nacional para el Conocimiento y Uso de la Biodiversidad.

Salazar, G. A. y Soto-Arenas, M. A. (2003). Stanhopea dodsoniana Salazar y Soto-Arenas. En E. Hágsater y M. A. Soto-Arenas (Eds.), Icones Orchidacearum 5-6. Orchids of Mexico 2-3 (p. 700). México, D. F.: Instituto Chinoín, A. C.

Salazar, G. A., Chávez-Rendón, C., Jiménez-Machorro, R. y de Ávila, A. (2011). A new species of Galeoglossum (Orchidaceae, Cranichidinae) from Oaxaca, Mexico. Systematic Botany, 36, 261-267.

Salazar, G. A., Hernández-Cardona, A. y Jiménez-Machorro, R. (2013). A new species of Deiregyne (Orchidaceae: Spiranthinae) from Hidalgo, Mexico. Botanical Sciences, 91, 129-133. 
Sánchez-Munguía, A. (2005). Uso del suelo agropecuario y deforestación en Tabasco 1950-2000. Villahermosa: Universidad Juárez Autónoma de Tabasco.

Semarnat (Secretaría de Medio Ambiente y Recursos Naturales). (2010). Norma Oficial Mexicana NOM-059-SEMARNAT-2010, Protección ambientalEspecies nativas de México de flora y fauna silvestres-Categorías de riesgo y especificaciones para su inclusión, exclusión o cambio-Lista de especies en riesgo. Diario Oficial de la Federación. 30 de diciembre de 2010. Segunda Sección. México.

Shorthouse, D. P. (2010). SimpleMappr, an online tool to produce publication-quality point maps. Recuperado el 9 septiembre, 2014 de: http://www.simplemappr.net

Solano-Gómez, R. (2010). Dos especies nuevas de Pleurothallidinae (Orchidaceae) de México. Lankesteriana, 9, 447-454.

Solano-Gómez, R., Jiménez-Machorro, R. y Damon, A. A. (2011). Two new records and one rediscovery for Orchidaceae of Mexico. Acta Botanica Mexicana, 96, 59-72.

Solano-Gómez, R. y Martínez-Ovando, E. (2011). Phragmipedium warscewiczii, a new record for the Mexican orchid flora. Revista Mexicana de Biodiversidad, 82, 69-75.
Soto-Arenas, M. A. (1986). Orquídeas de Bonampak, Chiapas. Orquídea (México), 10, 113-122.

Soto-Arenas, M. A. (1988). Listado actualizado de las orquídeas de México. Orquídea (México), 11, 233-272.

Soto-Arenas, M. A. (2003). Stanhopea oculata (G. Lodd.) Lindl. En E. Hágsater y M. A. Soto-Arenas (Eds.), Icones Orchidacearum 5-6. Orchids of Mexico 2-3 (p. 700). México, D. F.: Instituto Chinoín, A. C.

Soto-Arenas, M. A. y Salazar, G. A. (2007). Ficha técnica de Cycnoches ventricosum. En M. A. Soto-Arenas (Ed.), Información actualizada sobre las especies de orquídeas del PROY-NOM-059-ECOL-2000. México, D. F.: Instituto Chinoín, A. C., Herbario AMO. Bases de datos SNIB (Sistema Nacional de Información sobre Biodiversidad-Conabio (Comisión Nacional para el Conocimiento y Uso de la Biodiversidad). Proyecto N. ${ }^{\circ}$. W029.

Soto-Arenas, M. A. y Salazar, G. A. (2012). A new species of Deiregyne (Orchidaceae, Spiranthinae) from Mexico. Acta Botanica Mexicana, 101, 1-9.

Soto-Arenas, M. A., Hágsater, E., Jiménez, R., Salazar, G. A., Solano-Gómez, R., Flores, R., et al. (2007). Las orquídeas de México. Catálogo digital. México, D. F: Instituto Chinoín, A. C.

Tropicos. (2014). Missouri Botanical Garden. Recuperado el 9 septiembre, 2014 de: http://www.tropicos. 